\title{
Fatores associados a reações transfusionais imediatas em um hemocentro universitário: estudo analítico retrospectivo
}

\author{
Factors associated with immediate transfusion reactions in a university blood \\ center: an analytical, retrospective study
}

Vanessa Marques Vilar ${ }^{1}$ (D), Natany da Costa Ferreira² (D), Gislaine Rodrigues Nakasato ${ }^{3}$ (D), Fernando Luiz Lupinacci $^{4}$ (D), Juliana de Lima Lopes ${ }^{1}$ (D), Camila Takao Lopes ${ }^{1}$ (D)

\begin{abstract}
RESUMO
Estudo analítico, transversal, retrospectivo. Objetivos: Verificar a relação entre reações transfusionais imediatas, características demográficas e clínicas dos pacientes e características das hemotransfusões em um hemocentro universitário. Metodologia: O estudo foi realizado com dados de transfusões realizadas em 2016 no hemocentro da Universidade Federal de São Paulo. Foram coletados dados demográficos dos pacientes, características das hemotransfusões e ocorrência de reações transfusionais imediatas. Possíveis associações foram avaliadas usando os Testes de Fisher, Mann-Whitney, Qui-quadrado e $t$ de Student. Associações com valores de $p<0,05$ foram consideradas significativas. Resultados: Foram analisados dados de 320 fichas de pacientes, totalizando 938 transfusões. Dos produtos sanguíneos transfundidos, 90,3\% eram concentrados de hemácias, administrados por auxiliares de enfermagem em 43,8\% das situações. As reações transfusionais ocorreram em $4,3 \%$ das situações $(n=40)$. 0 sinal de reações mais frequente foi a febre $(47,5 \%)$, seguido de prurido $(22,5 \%)$. As reações foram mais prevalentes em pacientes com maior idade média $(p=0,0037)$, que receberam duas transfusões sanguíneas em comparação a uma $(p<0,0001)$, consequentemente mais bolsas de transfusões $(p=0,0002)$, maior volume total transfundido $(p<0,0001)$ e maior tempo de administração $(p=0,0017)$. Pacientes com anemia aplásica, leucemia mieloide aguda e neoplasias de órgãos sólidos tiveram reações transfusionais imediatas mais frequentemente do que pacientes com outros diagnósticos $(p \leq 0,05)$. Conclusões: $A$ ocorrência de reações transfusionais imediatas teve relação com a idade, diagnóstico médico, número de hemotransfusões, número de bolsas transfundidas, volume total transfundido e tempo de transfusão. O conhecimento desses fatores pode subsidiar treinamentos específicos voltados para a vigilância quanto às reações transfusionais imediatas.
\end{abstract}

Palavras-chave: Fatores de Risco; Reação Transfusional; Transfusão de Sangue.

\section{ABSTRACT}

An analytical, cross-sectional, retrospective study. Objectives: To investigate the relationship among immediate transfusion reactions, patient's demographic and clinical characteristics, and transfusion-related characteristics in a university blood center. Methodology: The study was performed with data regarding blood transfusions of the blood center at Universidade Federal de São Paulo in 2016. Patient's demographic and clinical characteristics and the characteristics of the immediate transfusion reactions were collected. Possible associations were evaluated using Fisher's exact test, Mann-Whitney, Chi-square, and Student's t-test. Associations with $\mathrm{p} \leq 0.05$ were considered statistically significant. Results: Data from 320 patient records were analyzed, totaling 938 blood transfusions. Of the blood products transfused, $90.3 \%$ were packed red blood cells administered by nursing assistants in $43.8 \%$ of the situations. Immediate transfusion reactions occurred in $4.3 \%$ of the situations $(n=40)$. The most common transfusion reaction was fever $(47.5 \%)$, followed by pruritus $(22.5 \%)$. The transfusion reactions were more frequent in older patients $(p=0.0037)$ who received two blood transfusions compared to one $(p<0.0001)$, consequently more transfusion bags $(p=0.0002)$, greater total transfused volume $(p<0.0001)$, and longer length of administration $(p=0.0017)$. Patients with aplastic anemia, acute myeloid leukemia, and solid organ neoplasms had immediate transfusion reactions more frequently than patients with other diagnoses $(p<0.05)$. Conclusion: The occurrence of immediate transfusion reactions was related to age, medical diagnosis, number of blood transfusions, number of transfused bags, total transfused volume, and length of administration. Knowledge of these factors may support specific training for immediate transfusion reactions surveillance.

Keywords: Risk Factors; Transfusion Reaction; Blood Transfusion.

1. Departamento de Enfermagem Clínica e Cirúrgica, Escola Paulista de Enfermagem, Universidade Federal de São Paulo (UNIFESP), São Paulo (SP), Brasil.

2. College of Nursing, University of Iowa, Iowa, IA, EUA.

3. Hospital do Coração, São Paulo (SP), Brasil.

4. Hemocentro, Escola Paulista de Medicina, UNIFESP, São Paulo (SP), Brasil.

$\triangle$ Camila Takao Lopes. Escola Paulista de Enfermagem. Rua Napoleão de Barros, 754, Sala 311 - Vila Clementino. CEP: 04024-002. São Paulo (SP), Brasil. ctlopes@unifesp.br | Recebido em: 21/01/2020 | Aprovado em: 14/07/2020 


\section{INTRODUÇÃO}

As hemotransfusões têm como finalidade o restabelecimento ou manutenção da capacidade de transporte de oxigênio, restauração do volume e/ou promoção da hemostasia sanguínea ${ }^{1}$. Nos Estados Unidos, aproximadamente 10,5 milhões de concentrados de hemácias, 2,3 milhões unidades de plasma e 2 milhões de concentrados de plaquetas foram transfundidos em 20172. Dados nacionais de 2016 estimaram aproximadamente 2,84 milhões de transfusões de sangue no Brasil, dentre os quais, 1,72 milhão de concentrado de hemácias, 500,6 mil concentrado de plaquetas e 350,4 mil bolsas de plasma fresco, dentre outros ${ }^{3}$.

Apesar de ser um tratamento que pode reduzir desfechos clínicos indesejáveis, como mortalidade, novo episódio de sangramento, infarto agudo do miocárdio, tromboembolismo venoso, acidente vascular encefálico ${ }^{4}$ e outros, a hemotransfusão pode acarretar reações transfusionais (RT) antes, durante e após o procedimento, relacionadas a sérios eventos adversos, inclusive fatais ${ }^{5,6}$.

As RT são definidas pelo Ministério da Saúde (MS) como quaisquer intercorrências que ocorrem como consequência da transfusão sanguínea durante ou após a administração de hemocomponentes, classificadas como reações transfusionais imediatas (RTI) ou tardias. As primeiras ocorrem nas primeiras 24 horas após o início da transfusão e incluem reação febril não hemolítica, reação hemolítica aguda, reação alérgica, lesão pulmonar relacionada à transfusão, sobrecarga volêmica, contaminação bacteriana, hipotensão por inibidor de enzima conversora de angiotensina, hemólise não imune, hipocalcemia, embolia aérea e hipotermia7. No Brasil, em 2015, 10.547 RT foram notificadas, dentre as quais $99 \%$ foram classificadas como RTI ${ }^{8}$.

As reações tardias, por sua vez, ocorrem após o período de 24 horas a partir do início da hemotransfusão e podem incluir a aloimunização de eritrócitos, hemossiderose, aloimunização de antígenos leucocitários humanos, doenças infecciosas, reação do enxerto contra o hospedeiro, púrpura após transfusão e imunomodulação?. Com vistas a auxiliar os profissionais de serviços de saúde a notificar incidentes e eventos adversos relacionados ao uso de diversos produtos de vigilância sanitária, como as reações decorrentes de uma transfusão sanguínea, a Agência Nacional de Vigilância Sanitária (ANVISA) desenvolveu o Sistema Nacional de Notificações para a Vigilância Sanitária $\left(\right.$ NOTIVISA) ${ }^{9}$. No período de Maio a Dezembro de 2016, o Hospital São Paulo notificou ao NOTIVISA cerca de 1.548 RT, dentre as quais $99,0 \%$ foram caracterizadas como RTI ${ }^{10}$.

Para otimizar os cuidados no processo de hemotransfusão, atualmente alguns programas de gerenciamento de sangue do paciente, como o Patient Blood Management (PBM), vêm sendo implementados. Os PBM são abordagens sistemáticas, baseadas em evidências, focadas nos pacientes, que possibilitam a otimização de transfusões de produtos sanguíneos, como a disponibilidade desses produtos em tempo hábil, redução de hemotransfusões desnecessárias, de efeitos adversos relacionados à transfusão e custos hospitalares. Nesses programas, a equipe multiprofissional é a responsável pela implementação de medidas com vistas a garantir o uso racional e seguro de hemocomponentes ${ }^{11}$.

Nesse contexto, a identificação dos fatores associados à RT pode auxiliar no reconhecimento precoce e no manejo de RTI quando presentes, bem como aprimorar o desenvolvimento de medidas de prevenção com a equipe multiprofissional responsável pela assistência direta a pacientes submetidos ao processo de hemotransfusão. Assim, o objetivo deste estudo foi verificar a relação entre reações transfusionais imediatas, características demográficas e clínicas dos pacientes e características das hemotransfusões em um hemocentro universitário.

\section{MATERIAIS E MÉTODOS}

Trata-se de estudo quantitativo, analítico, transversal e retrospectivo, realizado no hemocentro da Universidade Federal de São Paulo. A amostra de conveniência foi composta por registros de pacientes cadastrados no hemocentro. Foram incluídos aqueles relacionados a pacientes com idade maior ou igual a 18 anos que receberam hemotransfusões no ano de 2016.

Dados de caracterização demográfica e clínica e dados relacionados à hemotransfusões foram coletados das fichas transfusionais por uma aluna 
do terceiro ano do curso de graduação em enfermagem sob supervisão de uma docente ao longo dos anos de 2017 e 2018.

$\mathrm{O}$ instrumento de coleta de dados foi composto por variáveis independentes de interesse, classificadas em: dados de identificação do paciente, idade (em anos completos), sexo (masculino ou feminino) e cor da pele (preta, branca, amarela, parda ou indígena), diagnóstico médico, tipo sanguíneo, tipo de hemocomponente/hemoderivado $(\mathrm{CH}$, concentrado de plaquetas, plasma fresco congelado-PFC, concentrado de granulócitos, crioprecipitado, sangue total, sangue total reconstituído ou outro ( $\mathrm{CH}$ associado a concentrado de plaquetas ou PFC), tempo de administração (em minutos), tempo de armazenamento do produto sanguíneo (em minutos), categoria profissional de quem administrou o hemocomponente/hemoderivado (enfermeiro, auxiliar de enfermagem, enfermeiro e/ou auxiliar de enfermagem).

A variável desfecho consistiu na ocorrência de RTI. Quando presentes, as RTI foram caracterizadas conforme classificação da reação (leve, moderada, grave ou óbito) e sinais e sintomas (ansiedade, calafrio, choque, cianose de extremidades, cianose labial, dispneia, dor abdominal, dor lombar, dor torácica, edema agudo de pulmão, eritema, febre, hemoglobinúria, hipertensão arterial, hipotensão arterial, icterícia, náuseas, pápulas, rouquidão, taquicardia, taquipneia, tosse, tremores, urticária, vômito ou outros) ${ }^{7}$.

A análise de dados foi feita no software Statistical Package for Social Science (SPSS), versão 20. As variáveis nominais foram descritas por estatística descritiva (frequências absolutas e relativas). As variáveis quantitativas foram descritas por medidas de tendências central e dispersão (média, desvio padrão, mediana e intervalo interquartílico). A relação entre as variáveis independentes e as RTI foi verificada pelos Testes de Fisher, Mann-Whitney, $\mathrm{X}^{2}$ e $t$ de Student. Valores de $\mathrm{p} \leq 0,05$ foram considerados significativos.

O projeto foi aprovado pelo Comitê de Ética em Pesquisa com Seres Humanos da Universidade Federal deSão Paulo (CAAE: 71264217.4.0000.5505; Parecer número 0787/2017). Foram seguidos os preceitos éticos preconizados pela Resolução 466/2012 do Conselho Nacional de Saúde do Ministério de Saúde do Brasil.

\section{RESULTADOS}

De 1236 registros de pacientes que realizaram hemotransfusões no ano de 2016, 320 foram selecionados e os dados de 938 transfusões sanguíneas foram analisados. Todas as transfusões foram alogênicas. No entanto, houve dados faltantes (não registrados nas fichas transfusionais) para diferentes variáveis, como sexo, cor, diagnóstico médico e grupo sanguíneo.

As características demográficas e clínicas desses pacientes são apresentadas na Tabela 1.

\section{Tabela 1}

Características demográficas e clínicas dos pacientes submetidos a hemotransfusões em hemocentro de uma universidade pública da região metropolitana de São Paulo, SP, $2016(n=320)$

\begin{tabular}{|c|c|}
\hline Variável & Medida \\
\hline Idade, anos [mediana (IQ)] & $51,8(18-92)$ \\
\hline \multicolumn{2}{|l|}{$\operatorname{Sexo}(n, \%)$} \\
\hline Feminino & $166(51,9)$ \\
\hline Masculino & $152(47,5)$ \\
\hline Informação não disponível & $2(0,6)$ \\
\hline \multicolumn{2}{|l|}{ Cor $(n, \%)$} \\
\hline Branca & $165(51,5)$ \\
\hline Parda & $93(29,1)$ \\
\hline Preta & $28(8,8)$ \\
\hline Informação não disponível & $26(8,1)$ \\
\hline Amarela & $7(2,2)$ \\
\hline Vermelha & $1(0,3)$ \\
\hline \multicolumn{2}{|l|}{ Diagnóstico de admissão (n,\%) } \\
\hline Neoplasia de órgãos sólidos & $110(34,4)$ \\
\hline Anemia falciforme & $73(22,8)$ \\
\hline Outros & $62(19,4)$ \\
\hline Talassemia & $24(7,5)$ \\
\hline Informação não disponível & $19(5,9)$ \\
\hline Anemia aplásica & $17(5,3)$ \\
\hline Leucemia mieloide aguda & $15(4,7)$ \\
\hline \multicolumn{2}{|l|}{ Tipo sanguíneo (n, \%) } \\
\hline Informação incompleta* & $110(34,4)$ \\
\hline $0+$ & $102(31,9)$ \\
\hline$A+$ & $67(20,9)$ \\
\hline $\mathrm{B}+$ & $19(5,9)$ \\
\hline O- & $10(3,1)$ \\
\hline B- & $5(1,6)$ \\
\hline$A B+$ & $5(1,6)$ \\
\hline$A-$ & $2(0,6)$ \\
\hline
\end{tabular}

*: Fator $A B O$ ou Rh indisponível. IQ: intervalo interquartílico. 
Os pacientes tinham idade entre 18 e 92 anos, com mediana de 51,8 , predominantemente do sexo feminino $(166 ; 51,9 \%)$, de cor branca $(165 ; 51,6 \%)$, diagnosticados com neoplasias de órgãos sólidos $(110 ; 34,4 \%)$ e não tinham informação completa acerca do fator $A B O$ ou Rh (110; $34,4 \%)$.

A Tabela 2 apresenta os tipos de produtos sanguíneos transfundidos e os profissionais responsáveis por esta atividade.

\section{Tabela 2}

Tipo de produto sanguíneo transfundido e categoria profissional responsável pela administração no hemocentro de uma universidade pública da região metropolitana de São Paulo, SP, Brasil, $2016(n=938)$

\begin{tabular}{|c|c|}
\hline Variável & n (\%) \\
\hline \multicolumn{2}{|l|}{ Produto sanguíneo } \\
\hline Concentrado de hemácias & $847(90,3)$ \\
\hline Concentrado de plaquetas & $37(4,0)$ \\
\hline $\begin{array}{l}\text { Concentrado de hemácias e } \\
\text { concentrado de plaquetas }\end{array}$ & $36(3,8)$ \\
\hline Plasma fresco congelado & $14(1,5)$ \\
\hline $\begin{array}{l}\text { Concentrado de hemácias e plasma } \\
\text { fresco congelado }\end{array}$ & $4(0,4)$ \\
\hline \multicolumn{2}{|l|}{$\begin{array}{l}\text { Categoria profissional de quem administrou } \\
\text { o produto sanguíneo }\end{array}$} \\
\hline Auxiliar de enfermagem & $411(43,8)$ \\
\hline Enfermeiro & $400(42,6)$ \\
\hline Enfermeiro e auxiliar de enfermagem & $124(13,2)$ \\
\hline Não informado & $3(0,3)$ \\
\hline
\end{tabular}

Dentre as 938 hemotransfusões, 90,3\% estavam relacionadas a $\mathrm{CH}$, administrado por auxiliares de enfermagem em $43,8 \%$ das situações.

O tempo médio de administração dos produtos sanguíneos foi de 153,5 minutos, com tempo mínimo 50 e máximo de 395 minutos. Em relação ao tempo de armazenamento do produto, o tempo médio foi de 9,1 dias, com tempo mínimo e máximo de 2 e 35 dias, respectivamente.

As RTI ocorreram em 4,3\% das hemotransfusões $(n=40)$. Vinte e cinco $(62,5 \%)$ das RTI foram classificadas e consideradas leves. As demais, 15 RTI $(37,5 \%)$, não foram classificadas. Os sinais, sintomas e outras situações clínicas relacionadas às RTI estão apresentadas na Tabela 3.

\section{Tabela 3}

Sinais, sintomas e outras situações clínicas de pacientes que apresentaram reações transfusionais imediatas no hemocentro de uma universidade pública da região metropolitana de São Paulo, SP, Brasil, $2016(n=64)$

\begin{tabular}{lc}
\hline Sinal/sintoma & $\mathbf{n}(\%)$ \\
\hline Febre & $19(47,5)$ \\
Prurido & $9(22,5)$ \\
Dispneia & $5(12,5)$ \\
Calafrio & $4(10,0)$ \\
Eritema & $4(10,0)$ \\
Queda de saturação de $\mathrm{O}_{2}$ & $4(10,0)$ \\
Dor abdominal & $3(7,5)$ \\
Cefaleia & $3(7,5)$ \\
Taquicardia & $2(5,0)$ \\
Êmese & $2(5,0)$ \\
Dor em membros inferiores & $2(5,0)$ \\
Ansiedade & $1(2,5)$ \\
Hipotensão arterial & $1(2,5)$ \\
Náusea & $1(2,5)$ \\
Pápulas & $1(2,5)$ \\
Tosse & $1(2,5)$ \\
Bradicardia & $1(2,5)$ \\
Mal estar & $1(2,5)$ \\
\hline
\end{tabular}

As associações entre as variáveis demográficas e clínicas dos pacientes, bem como das transfusões sanguíneas com as RTI são apresentadas na Tabela 4.

As RTI foram mais frequentes em pacientes com maior idade média e naqueles com anemia aplásica comparados aos pacientes com anemia falciforme $(p=0,0012)$ ou com talassemia $(p=0,0245)$. Também houve maior ocorrência de RTI entre pacientes com leucemia mieloide aguda comparados àqueles com anemia falciforme $(p<0,0001)$, com talassemia $(p=0,0006)$, com neoplasias de órgãos sólidos $(p=0,0471)$ e com outros diagnósticos $(p=0,0207)$.

Pacientes com neoplasias de órgãos sólidos tiveram maior ocorrência de RTI quando comparados àqueles com anemia falciforme $(p=0,0012)$ e com talassemia $(p=0,0471)$. Pacientes com outros diagnósticos apresentaram RTI mais frequentemente do que aqueles com anemia falciforme $(p=0,015)$. 


\section{Tabela 4}

Associações entre as características demográficas e clínicas dos pacientes e características das transfusões sanguíneas com as reações transfusionais imediatas no hemocentro de uma universidade pública da região metropolitana de São Paulo, SP, Brasil, 2016

\begin{tabular}{|c|c|c|c|}
\hline \multirow[b]{2}{*}{ Variável } & \multicolumn{2}{|c|}{ Ocorrência de reação } & \multirow[b]{2}{*}{ p-valor } \\
\hline & $\underset{(n=40)}{\operatorname{Sim}}$ & $\begin{array}{c}\text { Não } \\
(n=896)\end{array}$ & \\
\hline Idade em anos, média \pm DP & $54,8 \pm 17,7$ & $45,6 \pm 18,9$ & $0,0037^{M}$ \\
\hline \multicolumn{4}{|l|}{ Sexo, n $(\%)^{*}$} \\
\hline Masculino & $22(55,0)$ & $458(51,4)$ & \multirow{2}{*}{$0,776^{Q}$} \\
\hline Feminino & $18(45,0)$ & $433(48,6)$ & \\
\hline \multicolumn{4}{|l|}{ Cor da Pele ou Raça, n (\%)* } \\
\hline Branca & $22(56,4)$ & $386(44,3)$ & \multirow{6}{*}{$0,406^{F}$} \\
\hline Preta & $3(7,7)$ & $120(13,8)$ & \\
\hline Parda & $13(33,3)$ & $349(40,0)$ & \\
\hline Amarela & $1(2,6)$ & $15(1,7)$ & \\
\hline Indígena & $0(0)$ & $1(0,1)$ & \\
\hline Outra & $0(0)$ & $1(0,7)$ & \\
\hline \multicolumn{4}{|l|}{ Diagnóstico médico, n (\%)* } \\
\hline Anemia falciforme & $2(5,0)$ & $301(34,5)$ & \multirow{8}{*}{$<0,0001^{F}$} \\
\hline Talassemia & $3(7,5)$ & $176(20,2)$ & \\
\hline Anemia aplásica & $7(17,5)$ & $63(7,2)$ & \\
\hline Leucemia mieloide aguda & $8(20,0)$ & $29(3,3)$ & \\
\hline Neoplasias de órgãos sólidos & $11(27,5)$ & $141(16,2)$ & \\
\hline Síndrome Blackfan-Diamond & $0(0)$ & $4(0,5)$ & \\
\hline Trombastenia de Glanzmann & $0(0)$ & $4(, 05)$ & \\
\hline Outros & $9(22,5)$ & $155(17,8)$ & \\
\hline \multicolumn{4}{|l|}{ Grupo sanguíneo, n (\%)* } \\
\hline$A$ & $16(47,1)$ & $289(39,3)$ & \multirow{4}{*}{$0,1941^{F}$} \\
\hline B & $1(2,9)$ & $104(14,1)$ & \\
\hline $\mathrm{O}$ & $16(47,1)$ & $328(44,6)$ & \\
\hline$A B$ & $1(2,9)$ & $15(2,0)$ & \\
\hline \multicolumn{4}{|l|}{ Fator $\mathrm{RH}, \mathrm{n}(\%)$} \\
\hline Positivo & $30(90,9)$ & $670(91,8)$ & \multirow{2}{*}{$0,7481^{F}$} \\
\hline Negativo & $3(9,1)$ & $60(8,2)$ & \\
\hline \multicolumn{4}{|l|}{ Número de transfusões em 2016,' n (\%) } \\
\hline Uma & $27(67,5)$ & $896(97,1)$ & \multirow{2}{*}{$<0,0001^{F}$} \\
\hline Duas & $13(32,5)$ & $27(2,9)$ & \\
\hline \multicolumn{4}{|l|}{ Tipo de produto, $\mathrm{n}(\%)$} \\
\hline Concentrado de hemácias & $26(65,0)$ & $819(91,4)$ & \multirow{5}{*}{$<0,0001^{F}$} \\
\hline Concentrado de plaquetas & $0(0)$ & $37(4,1)$ & \\
\hline Plasma fresco congelado & $1(2,5)$ & $13(1,5)$ & \\
\hline Concentrado de hemácias e concentrado de plaquetas & $12(30,0)$ & $24(2,7)$ & \\
\hline Concentrado de hemácias e plasma fresco congelado & $1(2,5)$ & $3(0,3)$ & \\
\hline Número de bolsas, média ( $\pm D P$ ) & $1,73( \pm 1,36)$ & $0,034( \pm 2,7)$ & $0,0002^{\top}$ \\
\hline Volume total em mL, média (DP) & $556,3( \pm 184,2)$ & $396,4( \pm 147,3)$ & $<0,0001^{\mathrm{M}}$ \\
\hline Tempo de administração em minutos média ( $\pm \mathrm{DP}$ ) & $204,2( \pm 93,9)$ & $151,6( \pm 65,9)$ & $0,0017^{\mathrm{M}}$ \\
\hline Armazenamento Bolsa 1 em dias, média ( $\pm D P$ ) & $8,3( \pm 5,0)$ & $8( \pm 5,0)$ & $0,6945^{\mathrm{M}}$ \\
\hline Armazenamento Bolsa 2 em dias, média ( $\pm D P$ ) & $8,2( \pm 5,7)$ & $7,9( \pm 4,7)$ & $0,9462^{\mathrm{M}}$ \\
\hline Armazenamento Bolsa 3 em dias, média ( $\pm D P$ ) & $8,5( \pm 2,1)$ & $6,5( \pm 6,4)$ & $0,749^{\mathrm{M}}$ \\
\hline \multicolumn{4}{|l|}{ Categoria profissional quem administrou, $\mathrm{n}(\%)$} \\
\hline Enfermeiro & $15(32,6)$ & $383(42,9)$ & \multirow{3}{*}{$0,214^{Q}$} \\
\hline Auxiliar de enfermagem & $15(32,6)$ & $395(44,2)$ & \\
\hline Enfermeiro e auxiliar de enfermagem & $16(34,8)$ & $12,9)$ & \\
\hline
\end{tabular}

*: Dados faltantes; DP: Desvio padrão; F: Teste de Fisher; M: teste de Mann-Whitney; Q: teste $x^{2}$; RH: Rhesus; T: teste $t$ de Student. 
Em relação ao número de transfusões sanguíneas, pacientes que receberam duas transfusões tiveram maior ocorrência de RTI quando comparados àqueles que receberam apenas uma transfusão $(p<0,0001)$. Também se associaram a maior ocorrência de RTI o maior número de bolsas $(p=0,0002)$, volume total transfundido $(p<0,0001)$ e maior tempo de administração $(p<0,0017)$.

Pacientes que receberam ambos $\mathrm{CH}$ e $\mathrm{CP}$ apresentaram RTI mais frequentemente do que aqueles que receberam apenas $\mathrm{CH}(p<0,0001)$ ou apenas $C P(p=0,0003)$.

\section{DISCUSSÃO}

O conhecimento acerca de procedimentos transfusionais, riscos relacionados ao manejo de hemocomponentes e eventos adversos decorrentes da transfusão sanguínea é essencial para garantir a qualidade da assistência de um hemocentro. A hemovigilância é uma ferramenta que auxilia no rastreio de potenciais riscos ao paciente submetido a processos transfusionais, bem como norteia o tratamento de eventos adversos. Na França, todos e quaisquer efeitos adversos relacionados à transfusões sanguíneas devem ser obrigatoriamente notificados ${ }^{12}$. No Brasil, o comitê transfusional de cada hospital, ambulatório e hemocentro deve monitorar, investigar e notificar todos os incidentes transfusionais imediatos e tardios ao sistema NOTIVISA ${ }^{9}$.

O presente estudo identificou que a prevalência de RTI no hemocentro estudado foi de 4,3\%, majoritariamente classificadas como reações leves. As RTI foram associadas à maior idade média, à transfusão de $\mathrm{CH}$ e $\mathrm{CP}$, maior número de hemocomponentes transfundidos e aos diagnósticos de anemia aplásica, anemia falciforme e talassemia. Em relação à gravidade e idade dos pacientes, dados nacionais de 2007 a 2015 corroboram nossos achados. A maioria das transfusões em âmbito nacional foram classificadas como leves $(81,3 \%$ a $85,9 \%)$ e quanto maior a idade, maior a ocorrência de $\mathrm{RT}^{8}$.

Estudo retrospectivo conduzido no Regional Blood Bank Transfusion Centre of North India analisou todos os eventos adversos relacionados às RT entre Janeiro de 2005 a Junho de 2013. Entre 160.973 unidades de hemocomponentes transfundidas pelo centro, $314(0,19 \%)$ RTI foram reportadas.
Os pacientes que tiveram RTI eram majoritariamente do sexo masculino (54\%), com idade média de 22,5 anos. Os eventos adversos mais identificados foram a reação transfusional febril não hemolítica $(54,7 \%)$, seguido por reações alérgicas $(41,4 \%)^{13}$.

A experiência de implementação de sistema de hemovigilância de um hospital na Arábia Saudita também reportou menor prevalência de RTI em comparação aos achados desse estudo. Entre 2012 e 2013, mais de 39 mil unidades de hemocomponentes foram transfundidos e as RTI foram reportadas em $0,2 \%$ dos pacientes, sendo majoritariamente reações alérgicas $(79,7 \%)$, seguido de reações febris $(17,9 \%)^{14}$.

Recentemente, um estudo retrospectivo analisou a incidência e a natureza das RT adversas de pacientes submetidos a hemotransfusões em clínicas, hospitais ambulatoriais, centros de diálise e domicílio, entre Janeiro de 2014 e Dezembro de 2016. Entre 3.284 transfusões sanguíneas, os autores excluíram as reações causadas por aloimunizações, inerentes do tratamento transfusional e identificaram $416(12,6 \%)$ reações adversas. Destas, $376(90,4 \%)$ ocorreram em pacientes ambulatoriais. A reação transfusional febril não hemolítica foi o evento adverso mais notificado (28,6\%), seguido pela reação alérgica responsável por $26,9 \%$ dos eventos. Os autores destacaram que a maioria dos casos foram notificados em pacientes no contexto ambulatorial, apesar da alta incidência de reações adversas relacionadas à hemotransfusão ${ }^{15}$.

Ainda que o hemocentro em estudo tenha obtido maiores taxas de RTI ao ser comparado aos resultados de outras publicações no âmbito hospitalar, destaca-se que o hemocentro em questão atende pacientes que necessitam de múltiplas transfusões de produtos sanguíneos ao longo da vida, com maior probabilidade de desenvolvimento de aloimunização, por exemplo.

A anemia aplásica, um dos diagnósticos médicos identificados na amostra desse estudo, é uma rara doença caracterizada pela produção insuficiente de glóbulos vermelhos, glóbulos brancos e plaquetas pela medula óssea, podendo afetar pessoas de qualquer idade e sexo. 0 alívio dos sintomas da doença é alcançado por meio de frequentes transfusões sanguíneas. Nos pacientes com anemia aplásica grave, as transfusões podem ser necessárias mais de uma vez por semana ${ }^{16}$. 
Por sua vez, na anemia falciforme, o principal componente do tratamento é a transfusão de $\mathrm{CH}^{17,18}$. Devido à grande demanda de transfusões sanguíneas para o tratamento dessa patologia, os pacientes podem desenvolver aloimunização de anticorpos devido à frequência de liberação de fatores pró-inflamatórios nas reações imunes desenvolvidas nas transfusões sanguíneas ${ }^{19}$.

A talassemia é outra condição médica que tem a terapia transfusional como componente-chave do tratamento. Assim como na anemia falciforme, diversas transfusões são realizadas e podem induzir a aloimunização. No caso da talassemia, a alta demanda transfusional está relacionada à ineficácia da eritropoiese ${ }^{20}$.

A frequência de RTI nos pacientes com talassemia no hemocentro estudado foi menor quando comparada aos achados de um estudo conduzido com 407 pacientes norte-americanos portadores de talassemia e que receberam transfusão de $\mathrm{CH}$. No estudo americano, os pacientes eram predominantemente mais jovens, com idade média de 22,3 $\pm 13,2$ anos. Quase metade dos sujeitos (48,0\%) apresentaram RT, incluindo reações alérgicas $(52,0 \%)$ e febris $(16,0 \%)$. Entre o grupo de pacientes que desenvolveram RT, 19,0\% eram pacientes aloimunizados ${ }^{21}$.

Nossos resultados são limitados por seu caráter retrospectivo, uma vez que os registros manuais nas fichas transfusionais podem não refletir fielmente a ocorrência das RTI, bem como suas características.

\section{CONCLUSÃO}

A ocorrência de reações transfusionais imediatas teve associação estatisticamente significativa com a idade, diagnóstico médico, número de transfusões sanguíneas, número de bolsas transfundidas, volume total transfundido e tempo de transfusão. O conhecimento desses fatores pode subsidiar treinamentos específicos voltados para a vigilância quanto às reações transfusionais imediatas.

\section{REFERÊNCIAS}

1. Turner ML. Safety of blood, blood derivatives, and plasma-derived products. Handb Clin Neurol. $2018 ; 153: 463-72$.
2. Jones JM, Sapiano MRP, Savinkina AA, Haass KA, Baker $M L$, Henry RA, et al. Slowing decline in blood collection and transfusion in the United States - 2017. Transfusion. 2020; 60(S2):S1-S9. doi: 10.1111/trf.15604

3. Brasil. Ministério da Saúde. Secretaria de Atenção à Saúde. Departamento de Atenção Especializada e Temática. Caderno de informação: sangue e hemoderivados: dados de 2016. [citado 2020 Jun 07]. Brasília (DF): Ministério da Saúde; 2018 Disponível em: http:// bvsms.saude.gov.br/bvs/publicacoes/caderno_informacao_sangue_hemoderivados_2016.pdf.

4. Carson JL, Guyatt G, Heddle NM, Grossman BJ, Cohn CS, Fung MK, et al. Clinical Practice Guidelines From the AABB: Red Blood Cell Transfusion Thresholds and Storage. JAMA. 2016;316(19):2025-35.

5. Frazier SK, Higgins J, Bugajski A, Jones AR, Brown MR. Adverse Reactions to Transfusion of Blood Products and Best Practices for Prevention. Crit Care Nurs Clin North Am. 2017;29(3):271-90.

6. Politis C, Wiersum JC, Richardson C, Robillard $P$, Jorgensen J, Renaudier $P$, et al. The International Haemovigilance Network Database for the Surveillance of Adverse Reactions and Events in Donors and Recipients of Blood Components: technical issues and results. Vox Sang. 2016;111(4): 409-17.

7. Brasil. Ministério da Saúde. Secretaria de Atenção à Saúde. Departamento de Atenção Especializada e Temática. Guia para uso de Hemocomponentes [Internet]. Brasília (DF); 2015 [citado 2019 Out 20]. Disponível em: http://bvsms.saude.gov.br/bvs/publicacoes/guia_uso_hemocomponentes_2ed.pdf.

8. Brasil. Agência Nacional de Vigilância Sanitária. Relatório de Hemovigilância 2015. [citado 2020 Jun 07]. Brasília (DF): Anvisa, 2016. Disponível em: http://portal.anvisa.gov.br/documents/33868/405589/ Hemovigil\%C3\%A2ncia+no+Brasil+-+Relat\%C3\%B3r io + consolidado $+2007+-+2015 / 51$ add6c1-0a15-4c189089-36a31e4cdd39.

9. Brasil. Ministério da Saúde. Gabinete do Ministro. Portaria n¹660, de 22 de julho de 2009. Institui o Sistema de Notificação e Investigação em Vigilância Sanitária-VIGIPOS, no âmbito do Sistema Nacional de Vigilância Sanitária, como parte integrante do Sistema Único de Saúde-SUS. Diário oficial da União, Brasília, 22 jul. 2009. [citado 2020 Junho 05]. Disponível em: http://bvsms.saude.gov.br/bvs/saudelegis/gm/2009/ prt1660_22_07_2009.html

10. Grandi JL, Grell MC, Areco KC, Barbosa DA. Hemovigilância: a experiência da notificação de reações transfusionais em Hospital Universitário. Rev Esc Enferm USP. 2018;52:e03331.

11. Mueller MM, Van Remoortel $H$, Meybohm P, Aranko K, Aubron C, Burger R, et al. Patient Blood Management: Recommendations From the 2018 Frankfurt Consensus Conference. JAMA. 2019;321(10):983-97.

12. Rieux C, Brittenham G, Bachir D, De Meyer E, Boudjedir K. French hemovigilance network. Delayed 
hemolytic transfusion reaction in the French hemovigilance system. Transfus Clin Biol. 2019;26(2):109-11.

13. Pahuja S, Puri V, Mahajan G, Gupta P, Jain M. Reporting adverse transfusion reactions: A retrospective study from tertiary care hospital from New Delhi, India. Asian J Transfus Sci. 2017;11(1):6-12.

14. Hindawi SI, Badawi MA, Raj ET, Gholam KA, Al-Weail So, Azher $F$. The use of transfusion quality indicators as a tool for hemovigilance system implementation at a tertiary care center in Saudi Arabia. Saudi Med J. 2016;37(5):538-43.

15. Moncharmont $\mathrm{P}$, Barday $\mathrm{G}$, Odent-Malaure $\mathrm{H}_{\mathrm{i}}$ Benamara $H$. Adverse transfusion reactions in recipients transfused in out-of-hospital. Transfus Clin Biol. $2018 ; 25(2): 105-8$.

16. Pickard AS, Huynh L, Ivanova JI, Totev T, Graham S, Mühlbacher AC, et al. Value of transfusion independence in severe aplastic anemia from patients' perspectives a discrete choice experiment. J Patient Rep Outcomes. $2017 ; 2(1): 13$.
17. Fasano RM, Booth GS, Miles M, Du L, Koyama T, Meier $E R$, et al. Red blood cell alloimmunization is influenced by recipient inflammatory state at time of transfusion in patients with sickle cell disease. $\mathrm{Br}$ J Haematol. $2015 ; 168(2): 291-300$.

18. Yazdanbakhsh K, Ware RE, Noizat-Pirenne F. Red blood cell alloimmunization in sickle cell disease: pathophysiology, risk factors, and transfusion management. Blood. 2012;120(3):528-37.

19. Bajpai M, Gupta S, Jain P. Alloimmunization in multitransfused liver disease patients: Impact of underlying disease. Asian J Transfus Sci. 2016;10(2):136-9.

20. Fasano RM, Chou ST. Red Blood Cell Antigen Genotyping for Sickle Cell Disease, Thalassemia, and Other Transfusion Complications. Transfus Med Rev. 2016;30(4):197-201. Review.

21. Vichinsky E, Neumayr L, Trimble S, Giardina PJ, Cohen AR, Coates $T$, et al. Transfusion complications in thalassemia patients: a report from the Centers for Disease Control and Prevention (CME). Transfusion. 2014; 54(4):972-81. 